\title{
On the Oscillation of Second-Order Nonlinear Neutral Delay Dynamic Equations on Time Scales
}

\author{
Quanxin Zhang, Xia Song, Li Gao \\ Department of Mathematics, Binzhou University, Shandong, China \\ Email: 3314744@163.com, songxia119@163.com, gaolibzxy@163.com
}

Received 9 May 2016; accepted 13 June 2016; published 16 June 2016

Copyright (C) 2016 by authors and Scientific Research Publishing Inc.

This work is licensed under the Creative Commons Attribution International License (CC BY).

http://creativecommons.org/licenses/by/4.0/

(c) (i) Open Access

\begin{abstract}
Based on Riccati transformation and the inequality technique, we establish some new sufficient conditions for oscillation of the second-order neutral delay dynamic equations on time scales. Our results not only extend and improve some known theorems, but also unify the oscillation of the second-order nonlinear delay differential equation and the second-order nonlinear delay difference equation on time scales. At the end of this paper, we give an example to illustrate the main results.
\end{abstract}

Keywords

Oscillation, Dynamic Equations, Neutral, Time Scale

\section{Introduction}

The theory of time scales was first proposed by Hilger [1] in order to unify continuous and discrete analysis. Several researchers have made greater contributions to various aspects of this new theory; see [2]-[4]. The new theory of dynamic equations on time scales not only unifies the theories of differential equations and difference equations, but also extends these classical cases to cases "in between", e.g., to so-called $q$-difference equations where $\mathbb{T}=q^{\mathbb{N}_{0}}=\left\{q^{t}: t \in \mathbb{N}_{0}\right.$ for $\left.q>1\right\}$.

In recent years, there has been much research involving the oscillation and nonoscillation of solutions of various equations on time scales such as [5]-[18]. In this paper we study and give the sufficient conditions for oscillation of the second-order neutral delay dynamic equation

$$
\left(a(t)\left([x(t)+r(t) x(\tau(t))]^{\Delta}\right)^{\gamma}\right)^{\Delta}+q(t) f(x(\tau(t)))=0, \quad t \in\left[t_{0}, \infty\right)_{\mathbb{T}},
$$

How to cite this paper: Zhang, Q.X., Song, X. and Gao, L. (2016) On the Oscillation of Second-Order Nonlinear Neutral Delay Dynamic Equations on Time Scales. Journal of Applied Mathematics and Physics, 4, 1080-1089.

http://dx.doi.org/10.4236/jamp.2016.46112 
where $t_{0}>0$ and $\mathbb{T}$ is unbounded time scale. Besides that, we will have hypotheses as follows throughout the paper:

$\left(\mathrm{H}_{1}\right) \quad \gamma$ is the ratio of two positive odd integers and $\gamma \geq 1$.

$\left(\mathrm{H}_{2}\right) \quad a, r, q: \mathbb{T} \rightarrow \mathbb{R}$ are positive rd-continuous functions with $r(t)$ satisfying $0 \leq r(t)<1$.

$\left(\mathrm{H}_{3}\right) \quad \tau \in \mathrm{C}_{r d}^{1}\left(\left[t_{0}, \infty\right)_{\mathbb{T}}, \mathbb{T}\right)$ is a strictly increasing and differentiable function such that $\tau(t) \leq t, \tau(t) \rightarrow \infty$ as $t \rightarrow \infty$ and $\tilde{\mathbb{T}}:=\tau(\mathbb{T}) \subset \mathbb{T}$.

$\left(\mathrm{H}_{4}\right) \quad f \in \mathrm{C}(\mathbb{R}, \mathbb{R})$ is a continuous function which satisfies $\frac{f(x)}{x^{\gamma}} \geq L$ for all $x \neq 0$, where $L$ is a positive constant.

In addition, for the sake of clearness and convenience,we will use the notation

$$
z(t):=x(t)+r(t) x(\tau(t))
$$

in the following narrative.

It is well known by reserchers in this field that an dynamic equation is called oscillatory in case all its solutions are oscillatory, and a solution of the equation is said to be oscillatory if it is neither eventually positive nor eventually negative. We only discuss those solutions $x$ of Equation (1.1) that are not eventually zero in this paper. Moreover we refer to [3] [4] for general basic background, ideas and more details on dynamic equations.

Because of $a(t)>0$, we shall consider Equation (1.1) respectively based on the case

$$
\int_{t_{0}}^{\infty}\left(\frac{1}{a(t)}\right)^{\frac{1}{\gamma}} \Delta t=\infty,
$$

and the other case

$$
\int_{t_{0}}^{\infty}\left(\frac{1}{a(t)}\right)^{\frac{1}{\gamma}} \Delta t<\infty
$$

\section{Several Lemmas}

In this section, we present and prove three lemmas which play important roles in the proofs of the main results.

Lemma 1. ([16]) Assume that $\tau: \mathbb{T} \rightarrow \mathbb{R}$ is strictly increasing, $\tilde{\mathbb{T}}:=\tau(\mathbb{T}) \subset \mathbb{T}$ is a time scale and $\tau(\sigma(t))=\sigma(\tau(t))$. Let $x: \tilde{\mathbb{T}} \rightarrow \mathbb{R}$. If $\tau^{\Delta}(t)$ and $x^{\Delta}(\tau(t))$ exist for $t \in \mathbb{T}^{k}$, then $(x(\tau(t)))^{\Delta}$ exists, and

$$
(x(\tau(t)))^{\Delta}=x^{\Delta}(\tau(t)) \tau^{\Delta}(t) .
$$

Lemma 2. ([3]) Assume that $x$ is delta-differentiable and eventually positive or eventually negative, then

$$
\left((x(t))^{\gamma}\right)^{\Delta}=\gamma \int_{0}^{1}[h x(\sigma(t))+(1-h) x(t)]^{\gamma-1} x^{\Delta}(t) \mathrm{d} h .
$$

We give the below lemma and prove it similar to that of Q. Zhang and X. Song ([17], Lemma 3.5).

Lemma 3. Based on (1.2), assume that $\left(H_{1}\right)-\left(H_{4}\right)$ hold. If $x$ is an eventually positive solution of (1.1), there exists $t_{1} \in\left[t_{0}, \infty\right)_{\mathbb{T}}$ such that

$$
z^{\Delta}(t)=[x(t)+r(t) x(\tau(t))]^{\Delta}>0,\left(a(t)\left(z^{\Delta}(t)\right)^{\gamma}\right)^{\Delta}<0, t \in\left[t_{1}, \infty\right)_{\mathbb{T}} .
$$

Proof. Assume $x(t)$ is an eventually positive solution of (1.1). That is, there exists $t_{1} \in\left[t_{0}, \infty\right)_{\mathbb{T}}$ such that $x(t)>0$ and $x(\tau(t))>0$ for $t \in\left[t_{1}, \infty\right)_{\mathbb{T}}$. Because of $z(t)=x(t)+r(t) x(\tau(t))$ and $0 \leq r(t)<1$, we get $z(t)>0$ esaily for $t \in\left[t_{1}, \infty\right)_{\mathbb{T}}$. At the same time for $t \in\left[t_{1}, \infty\right)_{\mathbb{T}}$, from equation (1.1) we obtain that

$$
\left(a(t)\left(z^{\Delta}(t)\right)^{\gamma}\right)^{\Delta}<0
$$

so $a(t)\left(z^{\Delta}(t)\right)^{\gamma}$ is decreasing. From (2.4), we know that $z^{\Delta}(t)$ is either eventually positive or eventually 
negative. Now we assert that $z^{\Delta}(t)>0$.

Suppose to the contrary that there exits $t_{2} \in\left[t_{1}, \infty\right)_{\mathbb{T}}$ such that $z^{\Delta}(t)<0$ for all $t \in\left[t_{2}, \infty\right)_{\mathbb{T}}$. Because $a(t)\left(z^{\Delta}(t)\right)^{\gamma}$ is decreasing,

$$
a(t)\left(z^{\Delta}(t)\right)^{\gamma} \leq a\left(t_{2}\right)\left(z^{\Delta}\left(t_{2}\right)\right)^{\gamma}=-M^{\gamma}
$$

for $t \in\left[t_{2}, \infty\right)_{\mathbb{T}}$, where $M=\left[a\left(t_{2}\right)\right]^{\frac{1}{r}}\left|z^{\Delta}\left(t_{2}\right)\right|>0$. Based on the above inequality (2.5), we get

$$
z^{\Delta}(t) \leq-M\left[\frac{1}{a(t)}\right]^{\frac{1}{\gamma}}, t \in\left[t_{2}, \infty\right)_{\mathbb{T}} .
$$

After integrating the two sides of inequality (2.6) from $t_{2}$ to $t \in\left[t_{2}, \infty\right)_{\mathbb{T}}$, we have

$$
z(t) \leq z\left(t_{2}\right)-M \int_{t_{2}}^{t}\left[\frac{1}{a(s)}\right]^{\frac{1}{\gamma}} \Delta s, \quad t \in\left[t_{2}, \infty\right)_{\mathbb{T}} .
$$

When $t \rightarrow \infty$, we get $\lim z(t)=-\infty$ from (1.2) and the above (2.7), which is contradictory to $z(t)>0$. So the above hypothesis of $z^{\unlhd}(t)<0$ is false. In other words, we get $z^{\Delta}(t)>0$ for $t_{1} \in\left[t_{0}, \infty\right)_{\mathbb{T}}$. This completes the proof.

\section{Main Results}

Now we state and prove our main results in this section.

Theorem 1. Based on (1.2), assume that the conditions $\left(H_{1}\right)-\left(H_{4}\right)$ hold. If there exists a positive nondecreasing $\Delta$-differentiable function $\delta \in \mathrm{C}_{r d}^{1}\left(\left[t_{0}, \infty\right)_{\mathbb{T}}, \mathbb{R}\right)$ such that for every $T \in\left[t_{0}, \infty\right)_{\mathbb{T}}$

$$
\limsup _{t \rightarrow \infty} \int_{T}^{t}\left[L \delta(s) q(s)(1-r(\tau(s)))^{\gamma}-\eta^{\gamma}(\tau(s)) \delta^{\Delta}(s)\right] \Delta s=\infty
$$

where

$$
\eta(\tau(t))=\left(\int_{T}^{t}\left(\frac{1}{a(\tau(s))}\right)^{\frac{1}{\gamma}} \tau^{\Delta}(s) \Delta s\right)^{-1},
$$

then (1.1) is oscillatory on $\left[t_{0}, \infty\right)_{\mathbb{T}}$.

Proof. Assume that (1.1) has a nonoscillatory solution $x$ on $\left[t_{0}, \infty\right)_{\mathbb{T}}$. We may assume that $x(t)>0$ and $x(\tau(t))>0$ for all $t \in\left[t_{1}, \infty\right)_{\mathbb{T}}, t_{1} \in\left[t_{0}, \infty\right)_{\mathbb{T}}$. By the definition of $z(t)$, it follows $z(t)>0$. From $\left(\mathrm{H}_{3}\right)$ we know $\tau(t) \leq t$, by Lemma 3 we have $z^{\Delta}(t)>0$, so $z(\tau(t)) \leq z(t)$, and $0 \leq r(t)<1$, we obtain

$$
x(t)=z(t)-r(t) x(\tau(t)) \geq z(t)-r(t) z(\tau(t)) \geq(1-r(t)) z(t), \quad t \in\left[t_{1}, \infty\right)_{\mathbb{T}} .
$$

The proof that $x$ is eventually negative is similar. By Lemma 3 we have $z^{\Delta}(t)>0$ for all $t \in\left[t_{2}, \infty\right)_{\mathbb{T}}$, $t_{2} \in\left[t_{1}, \infty\right)_{\mathbb{T}}$, and by Lemma 1 and $\left(\mathrm{H}_{3}\right)$, there exists $T \in\left[t_{2}, \infty\right)_{\mathbb{T}}$ such that $(z(\tau(t)))^{\Delta}>0$ for all $t \in[T, \infty)_{\mathbb{T}}$. Using (2.2) and (2.3), we have

$$
\begin{aligned}
\left((z(\tau(t)))^{\gamma}\right)^{\Delta} & =\gamma \int_{0}^{1}[h(z(\sigma(\tau(t))))+(1-h) z(\tau(t))]^{\gamma-1}(z(\tau(t)))^{\Delta} \mathrm{d} h \\
& \geq \gamma \int_{0}^{1}[h(z(\tau(t)))+(1-h) z(\tau(t))]^{\gamma-1}(z(\tau(t)))^{\Delta} \mathrm{d} h \\
& =\gamma(z(\tau(t)))^{\gamma-1}(z(\tau(t)))^{\Delta} .
\end{aligned}
$$

$\mathbb{T}$ is unbounded above, which implies $\mathbb{T}^{k}=\mathbb{T}$. Furthermore, from Lemma 1 we get

$$
(z(\tau(t)))^{\Delta}=z^{\Delta}(\tau(t)) \tau^{\Delta}(t) .
$$


Thus, by $\left(\mathrm{H}_{3}\right)$,

$$
\left((z(\tau(t)))^{\gamma}\right)^{\Delta} \geq \gamma(z(\tau(t)))^{\gamma-1} z^{\Delta}(\tau(t)) \tau^{\Delta}(t)>0
$$

Next we define the function $W(t)$ by

$$
W(t)=\delta(t) \frac{a(t)\left(z^{\Delta}(t)\right)^{\gamma}}{(z(\tau(t)))^{\gamma}}, t \in[T, \infty)_{\mathbb{T}} .
$$

Then on $[T, \infty)_{\mathbb{T}}$, we have $W(t)>0$. From the basic knowledge of the time scale calculus that you can see in [3], we obtain

$$
W^{\Delta}(t)=\frac{\delta(t)}{(z(\tau(t)))^{\gamma}}\left(a(t)\left(z^{\Delta}(t)\right)^{\gamma}\right)^{\Delta}+a(\sigma(t))\left(z^{\Delta}(\sigma(t))\right)^{\gamma} \frac{(z(\tau(t)))^{\gamma} \delta^{\Delta}(t)-\delta(t)\left((z(\tau(t)))^{\gamma}\right)^{\Delta}}{(z(\tau(t)))^{\gamma}(z(\tau(\sigma(t))))^{\gamma}} .
$$

From (1.1) and $\left(\mathrm{H}_{4}\right)$, we get

$$
\left(a(t)\left(z^{\Delta}(t)\right)^{\gamma}\right)^{\Delta}=\left(a(t)\left([x(t)+r(t) x(\tau(t))]^{\Delta}\right)^{\gamma}\right)^{\Delta} \leq-q(t) f(x(\tau(t))),
$$

i.e.,

$$
\begin{aligned}
W^{\Delta}(t) \leq & -L q(t) \delta(t)\left(\frac{x(\tau(t))}{z(\tau(t))}\right)^{\gamma}+\frac{a(\sigma(t))\left(z^{\Delta}(\sigma(t))\right)^{\gamma} \delta^{\Delta}(t)}{(z(\tau(\sigma(t))))^{\gamma}} \\
& -\frac{\delta(t) a(\sigma(t))\left(z^{\Delta}(\sigma(t))\right)^{\gamma}\left((z(\tau(t)))^{\gamma}\right)^{\Delta}}{(z(\tau(t)))^{\gamma}(z(\tau(\sigma(t))))^{\gamma}} \\
& \leq-L q(t) \delta(t)(1-r(\tau(t)))^{\gamma}+\frac{a(\sigma(t))\left(z^{\Delta}(\sigma(t))\right)^{\gamma} \delta^{\Delta}(t)}{(z(\tau(\sigma(t))))^{\gamma}} \\
& \leq-L q(t) \delta(t)(1-r(\tau(t)))^{\gamma}+\frac{a(\tau(t))\left(z^{\Delta}(\tau(t))\right)^{\gamma} \delta^{\Delta}(t)}{(z(\tau(t)))^{\gamma}} \\
& =-L q(t) \delta(t)(1-r(\tau(t)))^{\gamma}+a(\tau(t)) \delta^{\Delta}(t)\left(\frac{z^{\Delta}(\tau(t))}{z(\tau(t))}\right)^{\gamma} .
\end{aligned}
$$

On the other hand, because

$$
\begin{aligned}
z(\tau(t)) & =z(\tau(T))+\int_{T}^{t} z^{\Delta}(\tau(s)) \tau^{\Delta}(s) \Delta s \\
& =z(\tau(T))+\int_{T}^{t}\left(\frac{1}{a(\tau(s))}\right)^{\frac{1}{\gamma}}(a(\tau(s)))^{\frac{1}{\gamma}} z^{\Delta}(\tau(s)) \tau^{\Delta}(s) \Delta s \\
& \geq\left(\int_{T}^{t}\left(\frac{1}{a(\tau(s))}\right)^{\frac{1}{\gamma}} \tau^{\Delta}(s) \Delta s\right)\left(a(\tau(t))\left(z^{\Delta}(\tau(t))\right)^{\gamma}\right)^{\frac{1}{\gamma}}
\end{aligned}
$$


we get

$$
\left(\frac{z^{\Delta}(\tau(t))}{z(\tau(t))}\right)^{\gamma} \leq \frac{1}{a(\tau(t))}\left(\int_{T}^{t}\left(\frac{1}{a(\tau(s))}\right)^{\frac{1}{\gamma}} \tau^{\Delta}(s) \Delta s\right)^{-\gamma}=\frac{\eta^{\gamma}(\tau(t))}{a(\tau(t))}, \quad t \in[T, \infty)_{\mathbb{T}} .
$$

Using (3.7) in (3.6), we have

$$
W^{\Delta}(t) \leq-L q(t) \delta(t)(1-r(\tau(t)))^{\gamma}+\eta^{\gamma}(\tau(t)) \delta^{\Delta}(t), \quad[T, \infty)_{\mathbb{T}} .
$$

At last, integrating (3.8) from $T$ to $t$, we obtain

$$
0<W(t) \leq W(T)-\int_{T}^{t}\left[L \delta(s) q(s)(1-r(\tau(s)))^{\gamma}-\eta^{\gamma}(\tau(s)) \delta^{\Delta}(s)\right] \Delta s,
$$

which creates a contradiction to (3.1). This completes the proof.

Remark 1. From Theorem 1, we can obtain different conditions for oscillation of all solutions of (1.1) with different choices of $\delta(t)$.

Next, we give the conditions that guarantee every solution of (1.1) oscillates when (1.3) holds.

Theorem 2. Based on (1.3), assume that the conditions $\left(H_{1}\right)-\left(H_{4}\right)$, (3.1) and (3.2) hold. If for every $T \in\left[t_{0}, \infty\right)_{\mathbb{T}}$

where

$$
\int_{T}^{\infty}\left[\frac{1}{a(s)} \int_{T}^{s} \theta^{\gamma}(u)(1-r(\tau(u)))^{\gamma} q(u) \Delta u\right]^{\frac{1}{\gamma}} \Delta s=\infty
$$

$$
\theta(t)=\int_{t}^{\infty}\left(\frac{1}{a(s)}\right)^{\frac{1}{\gamma}} \Delta s
$$

then (1.1) is oscillatory on $\left[t_{0}, \infty\right)_{\mathbb{T}}$.

Proof. Assume that (1.1) has a nonoscillatory solution $x$ on $\left[t_{0}, \infty\right)_{\mathbb{T}}$, then it is neither eventually positive nor eventually negative. Without loss of generality, we may assume that $x(t)>0$, then $x(\tau(t))>0$ for all $t \in\left[t_{1}, \infty\right)_{\mathbb{T}}, \quad t_{1} \in\left[t_{0}, \infty\right)_{\mathbb{T}}$, it follows $z(t)=x(t)+r(t) x(\tau(t))>0$ and

$$
x(t)=z(t)-r(t) x(\tau(t)) \geq z(t)-r(t) z(\tau(t)) \geq(1-r(t)) z(t), \quad t \in\left[t_{1}, \infty\right)_{\mathbb{T}} .
$$

The proof is similar when $x$ is eventually negative. Since $a(t)\left(z^{\Delta}(t)\right)^{\gamma}$ is decreasing for all $t \in[T, \infty)_{\mathbb{T}}$ and $T \in\left[t_{1}, \infty\right)_{\mathbb{T}}$, it is eventually of one sign and hence $z^{\Delta}(t)$ is eventually of one sign. So we shall distinguish the following two cases to discuss:

(I) $z^{\Delta}(t)>0$ for $t \geq T$; and

(II) $z^{\Delta}(t)<0$ for $t \geq T$.

Case $(I)$. The proof that $z^{\Delta}(t)$ is eventually positive is similar to that in Theorem 1 , so it is omitted here.

Case (II). For $s \geq t \geq T$, we have

$$
a(s)\left(-z^{\Delta}(s)\right)^{\gamma} \geq a(t)\left(-z^{\Delta}(t)\right)^{\gamma}
$$

then

$$
-z^{\Delta}(s) \geq\left(\frac{a(t)}{a(s)}\right)^{\frac{1}{\gamma}}\left(-z^{\Delta}(t)\right)
$$

Integrating (3.11) from $t(t \geq T)$ to $u(u \geq t)$ and letting $u \rightarrow \infty$, we have

$$
z(t) \geq\left[\int_{t}^{\infty}\left(\frac{1}{a(s)}\right)^{\frac{1}{\gamma}} \Delta s\right](a(t))^{\frac{1}{\gamma}}\left(-z^{\Delta}(t)\right)=-\theta(t) a^{\frac{1}{\gamma}}(t) z^{\Delta}(t), \quad t \in[T, \infty)_{\mathbb{T}},
$$


and thus

$$
(z(t))^{\gamma} \geq-(\theta(t))^{\gamma} a(t)\left(z^{\Delta}(t)\right)^{\gamma} \geq-(\theta(t))^{\gamma} a(T)\left(z^{\Delta}(T)\right)^{\gamma}=b(\theta(t))^{\gamma}, \quad t \in[T, \infty)_{\mathbb{T}},
$$

where $b=-a(T)\left(z^{\Delta}(T)\right)^{\gamma}>0$. Applying (3.12) to Equation (1.1), we find

$$
\begin{aligned}
-\left(a(t)\left(z^{\Delta}(t)\right)^{\gamma}\right)^{\Delta} & \geq L q(t)(x(\tau(t)))^{\gamma} \geq L q(t)(1-r(\tau(t)))^{\gamma}(z(\tau(t)))^{\gamma} \\
& \geq L q(t)(1-r(\tau(t)))^{\gamma}(z(t))^{\gamma} \geq b L \theta^{\gamma}(t) q(t)(1-r(\tau(t)))^{\gamma}, \quad t \in[T, \infty)_{\mathbb{T}} .
\end{aligned}
$$

Integrating (3.13) from $T$ to $t$, we have

Therefore,

$$
\begin{aligned}
-a(t)\left(z^{\Delta}(t)\right)^{\gamma} & \geq-a(T)\left(z^{\Delta}(T)\right)^{\gamma}+b L \int_{T}^{t} \theta^{\gamma}(s) q(s)(1-r(\tau(s)))^{\gamma} \Delta s \\
& \geq b L \int_{T}^{t} \theta^{\gamma}(s) q(s)(1-r(\tau(s)))^{\gamma} \Delta s .
\end{aligned}
$$

$$
-z^{\Delta}(t) \geq\left[\frac{b L}{a(t)} \int_{T}^{t} \theta^{\gamma}(s)(1-r(\tau(s)))^{\gamma} q(s) \Delta s\right]^{\frac{1}{\gamma}} .
$$

Next integrating (3.14) from $T$ to $t$, we obtain

$$
-z(t)+z(T) \geq \int_{T}^{t}\left[\frac{b L}{a(s)} \int_{T}^{s} \theta^{\gamma}(u)(1-r(\tau(u)))^{\gamma} q(u) \Delta u\right]^{\frac{1}{\gamma}} \Delta s \rightarrow \infty, \quad t \rightarrow \infty .
$$

By (3.9), we have $\lim _{t \rightarrow \infty} z(t)=-\infty$, which contradicts $z(t)>0$. This completes the proof.

Remark 2. By Theorem 2, we get the sufficient condition of oscillation for Equation (1.1) when the condition (1.3) is satisfied, while the usual result existing is that the conditions (1.3) was established, then every solution of the Equation (1.1) is either oscillatory or converges to zero on $\left[t_{0}, \infty\right)_{\mathbb{T}}$.

Theorem 3. Based on (1.2), assume $\left(H_{1}\right)-\left(H_{4}\right)$ hold and $\tau(\sigma(t))=\sigma(\tau(t))$. If there exists a positive $\Delta$ differentiable function $\delta \in \mathrm{C}_{r d}^{1}\left(\left[t_{0}, \infty\right)_{\mathbb{T}}, \mathbb{R}\right)$ such that for every $T \in\left[t_{0}, \infty\right)_{\mathbb{T}}$

$$
\limsup _{t \rightarrow \infty} \int_{T}^{t}\left[L q(s) \delta(s)(1-r(\tau(s)))^{\gamma}-\frac{(a(\tau(s)))^{\frac{1}{\gamma}}\left(\delta^{\Delta}(s)\right)^{2}}{4 \gamma \delta(s) \tau^{\Delta}(s)}\left(\eta^{\sigma}(\tau(s))\right)^{\gamma-1}\right] \Delta s=\infty,
$$

where $\eta$ is as the same as that in (3.2), then (1.1) is oscillatory on $\left[t_{0}, \infty\right)_{T}$.

Proof. Assume that (1.1) has a nonoscillatory solution $x$ on $\left[t_{0}, \infty\right)_{\mathbb{T}}$. Without loss of generality, we can assume that $x(t)>0$ and $x(\tau(t))>0$ for all $t \in\left[t_{1}, \infty\right)_{\mathbb{T}}, t_{1} \in\left[t_{0}, \infty\right)_{\mathbb{T}}$. By the definition of $z(t)$, it follows $z(t)>0$. The proof when $\mathrm{x}$ is eventually negative is similar. Proceeding as the proof of Theorem 1 , we obtained (3.3) and (3.5). Using (3.3) in (3.5), we have that on $[T, \infty)_{\mathbb{T}}$

$$
W^{\Delta}(t) \leq-L q(t) \delta(t)(1-r(\tau(t)))^{\gamma}+\frac{\delta^{\Delta}(t)}{\delta(\sigma(t))} W(\sigma(t))-\frac{\gamma \delta(t) a(\sigma(t))\left(z^{\Delta}(\sigma(t))\right)^{\gamma} z^{\Delta}(\tau(t)) \tau^{\Delta}(t)}{(z(\tau(\sigma(t))))^{\gamma+1}}
$$

Also, since $\left(a(t)\left(z^{\Delta}(t)\right)^{\gamma}\right)^{\Delta}<0$, we have

i.e.,

$$
a(\tau(t))\left(z^{\Delta}(\tau(t))\right)^{\gamma} \geq a(\sigma(t))\left(z^{\Delta}(\sigma(t))\right)^{\gamma},
$$

$$
z^{\Delta}(\tau(t)) \geq \frac{(a(\sigma(t)))^{\frac{1}{\gamma}}}{(a(\tau(t)))^{\frac{1}{\gamma}}} z^{\Delta}(\sigma(t))
$$


Substituting (3.17) into (3.16), we obtain on $[T, \infty)_{\mathbb{T}}$

$$
W^{\Delta}(t) \leq-L q(t) \delta(t)(1-r(\tau(t)))^{\gamma}+\frac{\delta^{\Delta}(t)}{\delta(\sigma(t))} W(\sigma(t))-\frac{\gamma \delta(t) \tau^{\Delta}(t)}{(a(\tau(t)))^{\frac{1}{\gamma}}(\delta(\sigma(t)))^{\frac{\gamma+1}{\gamma}}}(W(\sigma(t)))^{\frac{\gamma+1}{\gamma}} .
$$

i.e.,

$$
\begin{aligned}
W^{\Delta}(t) \leq & -L q(t) \delta(t)(1-r(\tau(t)))^{\gamma}+\frac{\delta^{\Delta}(t)}{\delta(\sigma(t))} W(\sigma(t)) \\
& -\frac{\gamma \delta(t) \tau^{\Delta}(t)}{(a(\tau(t)))^{\frac{1}{\gamma}}(\delta(\sigma(t)))^{\frac{\gamma+1}{\gamma}}}(W(\sigma(t)))^{\frac{1-\gamma}{\gamma}}(W(\sigma(t)))^{2} .
\end{aligned}
$$

Now using inequality (3.7), we get

$$
\begin{aligned}
z(\tau(t)) & \geq\left(\int_{T}^{t}\left(\frac{1}{a(\tau(s))}\right)^{\frac{1}{\gamma}} \tau^{\Delta}(s) \Delta s\right)\left(a(\tau(t))\left(z^{\Delta}(\tau(t))\right)^{\gamma}\right)^{\frac{1}{\gamma}}, \\
& \geq\left(\int_{T}^{t}\left(\frac{1}{a(\tau(s))}\right)^{\frac{1}{\gamma}} \tau^{\Delta}(s) \Delta s\right)\left(a(t)\left(z^{\Delta}(t)\right)^{\gamma}\right)^{\frac{1}{\gamma}} .
\end{aligned}
$$

Hence, we have

$$
\frac{z(\tau(t))}{z^{\Delta}(t)} \geq \frac{a^{\frac{1}{\gamma}}(t)}{\eta(\tau(t))}
$$

This implies that on $[T, \infty)_{\mathbb{T}}$

$$
(W(t))^{\frac{1-\gamma}{\gamma}}=(\delta(t) a(t))^{\frac{1-\gamma}{\gamma}}\left(\frac{z(\tau(t))}{z^{\Delta}(t)}\right)^{\gamma-1} \geq(\delta(t) a(t))^{\frac{1-\gamma}{\gamma}} \frac{a^{\frac{\gamma-1}{\gamma}}(t)}{\eta^{\gamma-1}(\tau(t))}=\delta^{\frac{1-\gamma}{\gamma}}(t) \eta^{1-\gamma}(\tau(t)) .
$$

Using (3.19) in (3.18), we have on $[T, \infty)_{\mathbb{T}}$ that

$$
\begin{aligned}
W^{\Delta}(t) \leq & -L q(t) \delta(t)(1-r(\tau(t)))^{\gamma}+\frac{\delta^{\Delta}(t)}{\delta(\sigma(t))} W(\sigma(t)) \\
& -\frac{\gamma \delta(t) \tau^{\Delta}(t)}{(a(\tau(t)))^{\frac{1}{\gamma}}(\delta(\sigma(t)))^{2}}(\eta(\sigma(\tau(t))))^{1-\gamma}(W(\sigma(t)))^{2} \\
= & -L q(t) \delta(t)(1-r(\tau(t)))^{\gamma}+\frac{(a(\tau(t)))^{\frac{1}{\gamma}}\left(\delta^{\Delta}(t)\right)^{2}}{4 \gamma \delta(t) \tau^{\Delta}(t)}(\eta(\sigma(\tau(t))))^{\gamma-1} \\
& -\left[\frac{\sqrt{\gamma \delta(t) \tau^{\Delta}(t)(\eta(\sigma(t)))^{1-\gamma}}}{\delta} W(\sigma(t))-\frac{\sqrt{(a(\tau(t)))^{\frac{1}{\gamma}}} \delta^{\Delta}(t)}{2 \sqrt{\gamma \delta(t) \tau^{\Delta}(t)(\eta(\sigma(\tau(t))))^{1-\gamma}}}\right] \\
\leq & \left.-\left[L(t) \sqrt{(a(\tau(t)))^{\frac{1}{\gamma}}}\right]^{2} \delta(t)(1-r(\tau(t)))^{\gamma}-\frac{(a(\tau(t)))^{\frac{1}{\gamma}}\left(\delta^{\Delta}(t)\right)^{2}}{4 \gamma \delta(t) \tau^{\Delta}(t)}(\eta(\sigma(\tau(t))))^{\gamma-1}\right] .
\end{aligned}
$$


Integrating both sides of this inequality from $T$ to $t$, taking the limsup of the resulting inequality as $t \rightarrow \infty$ and applying condition (3.15), we obtain a contradiction to the fact that $W(t)>0$ for $t \in[T, \infty)_{\mathbb{T}}$. This completes the proof.

Using the same ideas as in the proof of Theorem 2, we can now obtain the following result based on (1.3).

Theorem 4. Under the condition (1.3), assume that the conditions $\left(H_{1}\right)-\left(H_{4}\right),(3.9)$ and (3.15) hold, then (1.1) is oscillatory on $\left[t_{0}, \infty\right)_{\mathbb{T}}$.

\section{Application}

Now we shall reformulate the above conditions which are sufficient for the oscillation of (1.1) when (1.2) holds on different time scales:

If $\mathbb{T}=\mathbb{R}$, Equation (1.1) becomes

$$
\left(a(t)\left(z^{\prime}(t)\right)^{\gamma}\right)^{\prime}+q(t) f(x(\tau(t)))=0, t \in\left[t_{0}, \infty\right),
$$

and then conditions (3.1) and (3.15), respectively, become

$$
\limsup _{t \rightarrow \infty} \int_{t_{1}}^{t}\left[L \delta(s) q(s)(1-r(\tau(s)))^{\gamma}-\delta^{\prime}(s)\left(\int_{\tau\left(t_{1}\right)}^{\tau(s)}\left(\frac{1}{a(u)}\right)^{\frac{1}{\gamma}} \mathrm{d} u\right)^{-\gamma}\right] \mathrm{d} s=\infty
$$

and

$$
\limsup _{t \rightarrow \infty} \int_{t_{1}}^{t}\left[L q(s)(1-r(\tau(s)))^{\gamma} \delta(s)-\frac{(a(\tau(s)))^{\frac{1}{\gamma}}\left(\delta^{\prime}(s)\right)^{2}}{4 \gamma \delta(s) \tau^{\prime}(s)}\left(\int_{\tau\left(t_{1}\right)}^{\tau \tau(s)}\left(\frac{1}{a(u)}\right)^{\frac{1}{\gamma}} \mathrm{d} u\right)^{1-\gamma}\right] \mathrm{d} s=\infty .
$$

The conditions (4.2) and (4.3) are new.

If $\mathbb{T}=\mathbb{Z}$, Equation (1.1) becomes

$$
\Delta\left(a_{n}\left(\Delta z_{n}\right)^{\gamma}\right)+q_{n} f\left(x_{n-\sigma}\right)=0, n=0,1,2, \cdots,
$$

and conditions (3.1) and (3.15), respectively, become

$$
\limsup _{n \rightarrow \infty} \sum_{l=n_{0}}^{n}\left[L q_{l} \delta_{l}\left(1-r_{l-\sigma}\right)^{\gamma}-\Delta \delta_{l}\left(\sum_{k=n_{0}-\sigma}^{l-\sigma-1}\left(\frac{1}{a_{k}}\right)^{\frac{1}{\gamma}}\right)^{-\gamma}\right]=\infty
$$

and

$$
\limsup _{n \rightarrow \infty} \sum_{l=n_{0}}^{n}\left[L q_{l} \delta_{l}\left(1-r_{l-\sigma}\right)^{\gamma}-\frac{\left(a_{l-\sigma}\right)^{\frac{1}{\gamma}}\left(\Delta \delta_{l}\right)^{2}}{4 \gamma \delta_{l}}\left(\sum_{k=n_{0}-\sigma}^{l-\sigma}\left(\frac{1}{a_{k}}\right)^{\frac{1}{\gamma}}\right)^{1-\gamma}\right]=\infty .
$$

At same time, the Theorems 1 and 3 are new for the case $\mathbb{T}=\mathbb{Z}$.

Example 1. Consider the second-order nonlinear delay 2-difference equations

$$
\left(t^{\frac{2}{3}}\left(z^{\Delta}(t)\right)^{\frac{5}{3}}\right)^{\Delta}+t^{-\frac{8}{5}}\left(x\left(\frac{t}{2}\right)\right)^{\frac{5}{3}}\left(1+x^{2}\left(\frac{t}{2}\right)\right)=0, t \in \overline{2^{\mathbb{Z}}}, t \geq t_{0}:=2,
$$

where $z(t)=x(t)+\frac{1}{2} x\left(\frac{t}{2}\right)$. This gives

$$
a(t)=t^{\frac{2}{3}}, q(t)=t^{-\frac{8}{5}}, f(x)=x^{\frac{5}{3}}\left(1+x^{2}\right), r(t)=\frac{1}{2}, \tau(t)=\frac{t}{2}, \gamma=\frac{5}{3} .
$$


The conditions $\left(\mathrm{H}_{1}\right)-\left(\mathrm{H}_{3}\right)$ are clearly satisfied, and $\left(\mathrm{H}_{4}\right)$ holds with $L=1$. Because

$$
\int_{2}^{t}\left(\frac{1}{a(s)}\right)^{\frac{1}{\gamma}} \Delta s=\int_{2}^{t} s^{-\frac{2}{5}} \Delta s=\frac{t^{\frac{3}{5}}-2^{\frac{3}{5}}}{2^{\frac{3}{5}}-1} \rightarrow \infty \text { as } t \rightarrow \infty, t \geq 2,
$$

(1.2) is satisfied. Now let $\delta(t)=t^{\frac{3}{5}}$ for all $t>s \geq 2$, and then

$$
\eta(\tau(t))=\left(\int_{2}^{t}\left(\frac{1}{a(\tau(s))}\right)^{\frac{1}{\gamma}} \tau^{\Delta}(s) \Delta s\right)^{-1}=\left(\frac{1}{2^{\frac{3}{5}}} \int_{2}^{t} s^{-\frac{2}{5}} \Delta s\right)^{-1}=2^{\frac{3}{5}} \frac{2^{\frac{3}{5}}-1}{t^{\frac{3}{5}}-2^{\frac{3}{5}}} .
$$

Thus when $t \rightarrow \infty$, we have

$$
\int_{2}^{t}\left[L \delta(s) q(s)(1-r(\tau(s)))^{\gamma}-\eta^{\gamma}(\tau(s)) \delta^{\Delta}(s)\right] \Delta s=\int_{2}^{t}\left[\frac{1}{2^{\frac{5}{3}} s}-2\left(\frac{2^{\frac{3}{5}}-1}{s^{\frac{3}{5}}-2^{\frac{3}{5}}}\right)^{\frac{5}{3}}\left(s^{\frac{3}{5}}\right)^{\Delta}\right] \Delta s \rightarrow \infty .
$$

It is easy to see that (3.1) is satisfied as well. Altogether, the Equation (4.7) is oscillatory by Theorem 1.

\section{Acknowledgements}

We thank the Editor and the referee for their comments. Research of Q. Zhang is funded by the Natural Science Foundation of Shandong Province of China grant ZR2013AM003. This support is greatly appreciated.

\section{References}

[1] Hilger, S. (1990) Analysis on Measure Chains-A Unified Approach to Continuous and Discrete Calculus. Results in Mathematics, 18, 18-56. http://dx.doi.org/10.1007/BF03323153

[2] Agarwal, R.P., Bohner, M., O’Regan, D. and Peterson, A. (2002) Dynamic Equations on Time Scales: A Survey. Journal of Computational and Applied Mathematics, 141, 1-26. http://dx.doi.org/10.1016/S0377-0427(01)00432-0

[3] Bohner, M. and Peterson, A. (2001) Dynamic Equations on Time Scales: An Introduction with Applications. Birkhäuser, Boston. http://dx.doi.org/10.1007/978-1-4612-0201-1

[4] Bohner, M. and Peterson, A. (2003) Advances in Dynamic Equations on Time Scales. Birkhäuser, Boston. http://dx.doi.org/10.1007/978-0-8176-8230-9

[5] Bohner, M. and Saker, S.H. (2004) Oscillation of Second Order Nonlinear Dynamic Equations on Time Scales. Rocky Mountain Journal of Mathematics, 34, 1239-1254. http://dx.doi.org/10.1216/rmjm/1181069797

[6] Erbe, L. (2001) Oscillation Criteria for Second Order Linear Equations on a Time Scale. Canadian Applied Mathematics Quarterly, 9, 345-375.

[7] Erbe, L., Peterson, A. and Rehak, P. (2002) Comparison Theorems for Linear Dynamic Equations on Time Scales. Journal of Mathematical Analysis and Applications, 275, 418-438. http://dx.doi.org/10.1016/S0022-247X(02)00390-6

[8] Sun, S., Han, Z. and Zhang, C. (2009) Oscillation of Second Order Delay Dynamic Equations on Time Scales. Journal of Applied Mathematics and Computing, 30, 459-468. http://dx.doi.org/10.1007/s12190-008-0185-6

[9] Zhang, Q., Gao, L. and Wang, L. (2011) Oscillation of Second-Order Nonlinear Delay Dynamic Equations on Time Scales. Computers \& Mathematics with Applications, 61, 2342-2348. http://dx.doi.org/10.1016/j.camwa.2010.10.005

[10] Grace, S.R., Bohner, M. and Agarwal, R.P. (2009) On the Oscillation of Second-Order Half-Linear Dynamic Equations. Journal of Difference Equations and Applications, 15, 451-460. http://dx.doi.org/10.1080/10236190802125371

[11] Erbe, L., Hassan, T.S. and Peterson, A. (2009) Oscillation Criteria for Nonlinear Functional Neutral Dynamic Equations on Time Scales. Journal of Difference Equations and Applications, 15, 1097-1116. http://dx.doi.org/10.1080/10236190902785199

[12] Agarwal, R.P., Bohner, M. and Saker, S.H. (2005) Oscillation of Second Order Delay Dynamic Equations. Canadian Applied Mathematics Quarterly, 13, 1-18.

[13] Sahiner, Y. (2005) Oscillation of Second Order Delay Differential Equations on Time Scales. Nonlinear Analysis: Theory, Methods \& Applications, 63, 1073-1080. http://dx.doi.org/10.1016/j.na.2005.01.062 
[14] Erbe, L., Peterson, A. and Saker, S.H. (2007) Oscillation Criteria for Second Order Nonlinear Delay Dynamic Equations. Journal of Mathematical Analysis and Applications, 333, 505-522. http://dx.doi.org/10.1016/j.jmaa.2006.10.055

[15] Saker, S.H. (2005) Oscillation Criteria of Second-Order Half-Linear Dynamic Equations on Time Scales. Journal of Computational and Applied Mathematics, 177, 375-387. http://dx.doi.org/10.1016/j.cam.2004.09.028

[16] Han, Z., Li, T., Sun, S. and Zhang, C. (2009) Oscillation for Second-Order Nonlinear Delay Dynamic Equations on Time Scales. Advances in Difference Equations, 2009, Article ID: 756171. http://dx.doi.org/10.1155/2009/756171

[17] Zhang, Q. and Song, X. (2014) On the Oscillation for Second-Order Half-Linear Neutral Delay Dynamic Equations on Time Scales. Abstract and Applied Analysis, 2014, Article ID: 321764. http://dx.doi.org/10.1155/2014/321764

[18] Zhang, Q. and Liu, S. (2016) Oscillation Criteria for Second-Order Nonlinear Delay Dynamic Equations on Times Scales. British Journal of Mathematics \& Computer Science, to Be Published.

\section{Submit or recommend next manuscript to SCIRP and we will provide best service for you:}

Accepting pre-submission inquiries through Email, Facebook, Linkedin, Twitter, etc A wide selection of journals (inclusive of 9 subjects, more than 200 journals)

Providing a 24-hour high-quality service

User-friendly online submission system

Fair and swift peer-review system

Efficient typesetting and proofreading procedure

Display of the result of downloads and visits, as well as the number of cited articles

Maximum dissemination of your research work

Submit your manuscript at: http://papersubmission.scirp.org/ 